nicht etwa einer späteren Umschmelzung unterworten worden sind. Thre Erstarrungsgeschichte hat sich jedenfalls ohne Gegenwart eines starken anisotropen Schwerefeldes abgespielt.

Im selben Sinne, wenngleich nicht derart kraß, kann das Auftreten von Troilit- (und Troilit-Ohromit-) Knollen in meteorisehem Nickeleisen gedeutet werden. Diese Knollen entsprechen der Sulfid-Oxyd-Schale im Erdinnern.

Es wäre von grobem Interesse, die Gesamtheit der Meteoriten auf den Grad ihrer Schweresonderung zu untersuchen, da man hieraus vielleicht $z u$ weiteren Aufschlüssen über ihre Herkunft und Geschichte gelangen könnte.

In manchen Eisenmeteoriten findet man ungleichmäBige Verteilung der leichteren Einschlüsse im Nickeleisen, wie lokale, unsymmetrische Anreicherung von Olivinkristallen, schlierige oder streifenformige Anordnung des Schwefeleisens, welches darauf hindeutet, daB gewisse, wenn auch schwache Schwerewirkungen ihren Einfluß geltend machten, während das Nickeleisen noch flüssig war ${ }^{6}$ ).

Von besonderem Interesse wäre es auch, die Erstarrungsgeschichte der Pallasite näher zu studieren, um insbesondere die Frage zu behandeln, in welchen Pallasiten die Silikattropfen bereits vor Erstarrung des Metallbades erstarrten, in welchen erst nachher, und ob diese Erstarrungsgeschichte in Beziehung zu den Schmelzdiagrammen Nickel-Eisen und Olivin-Fayalit gebracht werden kann.

In Meteoriten finden wir niemals Mineralkombinationen, welche der Eklogitschale der Erde entspreehen würden; dies hat offenbar seinen Grund darin, daß in Kleinen Himmelskörpern nicht der notwendige Belastungsdruck für die Bildung von Eklogiten aufgebracht werden kann.

Das Vorkommen von Diamant als Seltenheit in einzelnen Meteoreisen könnte zwar auf Bildungsbedingungen hindeuten, welche mit der Eklogitfacies vergleichbar sind, immerbin kann der Diamant auch ohne starken Belastungsdruck entstanden sein, in Analogle mit Moissans bekannter Synthese.

Das Auftreten von solchen Struktureigentümlichkeiten, die auf Abwesenheit starker Gravitationswirkungen hindeuten, bringt uns zu der Frage, ob es experimentell' gelingen könnte, solche Strukturen nachzuahmen, wie sie den Pallasiten zu eigen sind. Im Laboratorium oder im technischen Schmelzofen sind die Einwirkungen des Schwerefeldes nicht zu eliminieren, wir können keine dauernde Suspension von Silikattropfen in geschmolzenem Nickeleisen herstellen; es beruht ja die technische Herstellung von Eisen im Hochofen gerade auf der gravitativen Sonderung von Eisen und Silikatschlacke. Es bietet sich nur eine Möglichkeit, den EinfluB eines Schwerefeldes (bzw. Trügheitsfeldes) bei einem Schmelzversuch

\footnotetext{
6) Möglicherweise durch Rotation des Meteoriten (Zentrifugalkraft) hervargebracht.
}

weitgehend auszuschalten, nämlich den Versuch in einer frei fallenden Schmelzmasse auszufuhren, die genügend schwer ist, um die Hemmung durch Luftwiderstand weitgehend auszuschalten, etwa indem man die Schmelze in einen granatenförmigen Behälter von einem Flugzeug himabfallen liebe.

\section{Neue physiologische Untersuchungen über das Leben in den Anden.}

\author{
Fon A. Loewy, Berlin.
}

In der Nummer vom 29. Juli d. J. der englischen Zeitschrift Nature findet sich ein Aufsatz des bekannten Cambridger Physiologen J. Barcroft: ,The physiology of life in the Andes."

Er enthält in Kurzer Darstellung die Ergebnisse von Untersuchungen, die in den peruanischen Anden angestellt wurden. Sie betreffen die Besonderheiten im Ablauf der physiologischen Vorgänge, welche ein beschwerdefreies und körperliche Árbeit gestattendes Leben in jenen Hochregionen für die Eingeborenen ermöglichen, und die Anpassungsvorgänge, die bei Tiefländern sich allmählich ausbilden, wenn sie längere Zeit in diesen Hohen leben, und die dazu fuhren, dab auch diese, wenigstens bei Körperruhe, schließlich keinerlei Krankheitssymptome mehr zeigen.

Beim Lesen des Barcroftschen Berichtes muB denjenigen, der selbst in den Zeiten von Deutschland's Glanz und Ruhm an ähnlichen Unternehmungen sich beteiligt hat, ein Gefühl der Niedergesehlagenheit und des Neides beschleichen bei der Wahrnehmung, daB deutsche Eorscher heute und wohl noch für lange Zeit auBerstande sind, derartige Untersuchungen auch nur zu planen, während die angelsächsisehen Forscher schon kurz mach Beendigung des Weltkrieges in der Lage waren, an große wissenschaftliche Unternehmungen heranzugehen und Gegenstände weiterzufördern, an deren bisheriger Bearbeitung die deutsche Wissenschaft einen nicht geringen Anteil hatte.

Aus der Darstellung Barcrofts geht dieser Anteil allerdings nicht hervor, denn sie beschränkt sich auf die Beschreibung der eigenen Expedition und deren Ergebnisse. Letztere bestätigen vielfach mur die älteren Befunde. In einigen Punkten jedoch bringen sie neue und überraschende Kenntnisse.

Mit Unterstutzung verschiedener englischer und amerikanischer Institute und mit Hilfe von Spenden von privater Seite bildeten sich eine englische und eine amexikanische Abteilung, deren erste von Liverpool, deren zweite ron New York nach Lima aufbrach. Von dort ging die Reise mit der transandinischen Bahn über die Randkordillere in Höhe von $5300 \mathrm{~m}$ hinab nach Oroya $(4000 \mathrm{~m})$, das in den Steppen zwischen den beiden parallelen Kordillerenketten gelegen ist, und ron da nach dem Bestimmungsorte Cerro de 
Pasco (4500 m hoch). Dieser Ort hat verschiedene Vorzüge vor den bisher für physiologische Höhenuntersuchungen benutzten. In Europa kam in dieser Hinsicht bisher - von Untersuchungen in niedrigeren Höhenlagen abgesehen - der Monte Rosa in Betracht, an dessen Nordabiang Zuntz und Schumburg 1894 sowie später Kronecker ihre Untersuchungen anstellten, an dessen Südabhang und auf dessen zweiter Spitze (Gnifettispitze, deutsch Signalkuppe), von Süden herkommend, Mosso in den letzten Jahren des verflossenen Jahrhunderts in mehreren Expeditionen seine Forschungen durchführte. Auf Mossos Arbeitsgebiet betätigten sich später die Brüder Loewy und L. Zuntz, sodann N. Zuntz, Loewy, Müller, Caspari (1901), Zuntz and Durig 1905 sowie Fuchs und kurz vor dem Kriege Kestner mit mehreren Mitarbeitern. In allen diesen Untersuchungen wurde der Einfluß einer Höhe von $3900 \mathrm{~m}$ und $4560 \mathrm{~m}$ auf die physiologischen Vorgänge erforscht, sowohl am Menschen wie an verschiedenen Säugetieren.

Den gleichen Zwecken ist weiter dienstbar gemacht worden der Pic von Teneriffa, wo schon 1878 Marcet sich mit Untersuchungen über den respiratorischen Stoffwechsel betätigte, dann Zuntz in Gemeinschaft mit Durig, von Schrötter, Bareroft und Douglas. Endlich wurde der Pikes Peak in Colorado von einer Reihe englischer und amerikanischer Physiologen unter Führung von Haldane (Oxford) zu physiologisehen Untersuchungen aufgesucht.

Dieser langen Reihe von wissenschaftlichen Unternehmungen schließt sich nun die hier beschriebene an, an der (auBer Barcroft) I. C. Meakins aus Edinburgh, H. Doggart aus Cambridge, und von amerikanischer Seite Bock, Forbes (von der Harvard-Universität), Redfield (Toronto), George Harrop und C. Binger (New York) teilnahmen.

Cerro de Pasco wurde gewählt, weil es bequem besonders für iden Transport wissenschaftlicher Apparate zu erreichen ist, weil es trotz seiner beträontlichen Höhenlage in noch bewohnten Gegenden liegt, was den Uniterhalt der Mitglieder der Expedition sehr erleichtert, ferner weil reichlich Wasser vorhanden ist, was für die Ausführung der Untersuchungen wesentlich ist. Dabei gibt es in Cerro de Pasco einen alten, aus spanischer Zeit stammenden Bergwerksbetrieb, und mit Hilfe von dessen (englischen) Ingenieuren wurde ein Güterwagen als Laboratorium eingerichtet, das mit elektrischem Licht, elektrischer Kraft und Heizung ausgestattet wurde, und in dem selbst eine Einrichtung für Röntgenuntersuchungen angebracht wurde. Cerro de Paseo spielt uibrigens in den Reisebeschreibungen von Hoch-Peru seit langem eine Rolle. Schon in der ersten Hälfte des vorigen Jahrhunderts wurde es von Tschudi und Pöppig besucht, und Beschreibungen der Bergkrankheit dknüpfen sich an seinen Namen. Die Eingeborenen, von indianischer Abkunft, werden "Cholo" genannt, sind von kleiner Statur und blab oder doch nux wenig blutreich im Gesicht. Sie sind breit gebaut. Messungen des Brustumfanges ergaben eine ungewöhnlich hohe Zahl. Von Dreyer rühren Tabellen her, in denen die Beziehung $z$ wischen Brustumfang und Rumpflänge bei gesunden Europäern (Engländern) zusammengestellt sind. Entsprechend ihrer Rumpflänge hätte der Brustumfing der Cholo $79 \mathrm{~cm}$ betragen müssen, während er in Wirklichkeit $92 \mathrm{~cm}$ ausmachte. Bei den Expeditionsmitgliedern lag der Brustumfang nur wenig über dem nach der Rumpflänge zu erwartenden Werte. Der erhebliche Brustumfang von Hochgebirgsbewohnern ist schon früher erkannt worden. So berichtet darüber meines Wissens Jourdanet (Infl. de la pression de l'air etc., Paris 1875) nach Beobachtungen in Mexiko. Röntgenbilder des Brustkorbes zeigten nun, da. die Rippenstellung der Cholos von der der Tiefenbewohner abweicht. Der Ansatz der Rippen an der Wirbelsäule geschieht unter einem weniger spitzen Winkel, ihr Verlauf von der Wirbelsäule ab erfolgt mehr horizontal. Barcroft möchte dies als kompensatorisehen Vorgang ansehen, durch den das Blut befähigt werden soll, leichter Sauerstoff aufzunehmen, indem er darauf hinweist, daß im Tieflande Kranke, die an Lungenerweiterung oder anderen, mit Luftmangel einhergehenden Krankheiten leiden, eine ähnliche Form des Brustkastens zeigen. Interessant ist auch, daß bei den Eingeborenen in Cerro de Pasco nicht selten sogenannte ,Trommelschlägerfinger" gefunden werden, d. h. kolbige Verdickungen der Endglieder der Finger. Im Tieflande findet man sie nur unter krankhaften Verhältnissen, bei Zuständen, bei denen die Sauerstoffversorgung der extremen Körperteile unzureichend ist.

Soll die Sauerstoffarmut bzw. der abnorm niedrige Sauerstoffdruck der Höhenluft und die daraus sich ergebende Erschwerung der Sauerstoffversorgung des menschlichen und tierischen Körpers kompensier, werden, so läßt sich theoretisch eine ganze Reihe von physiologischen Vorgängen ableiten, die geeignet sind, die Sauerstoffversorgung der Körpergewebe zu verbessern. Aller dieser bedient sich die Natur nicht. So könnte ein beschleunigter Blutkreislauf zu einer vermehrten Sauerstoffzufuhr zu den Geweben führen und damit den Eintritt des Sauerstoffmangels hinausschieben. Jedoch tritt eine Blutstrombeschleunigung im Hochgebirge nicht ein. Dieser negative Befund von Barcroft und Genossen bestätigt, was schon vor. langer Zeit Loewy für den Aufenthalt im luftverdünnten Raum der pneumatischen Kammer in Versuchen an Hunden zeigen ksonnte (Respiration und Zirkulation usw., Berlin 1895) und was neuerlich mit anderer Methode ron Doi (Journal of Physiology 55, 1921) wieder festgestellt wurde.

Dagegen dienen der vermehrten Sauerstoffzufuhr Vorgänge, die die Verfasser in Überein- 
stimmung mit den Befunden der früheren Forscher in anderen Gebirgsgegenden feststellen konnten. Zunächst eine Steigerung der Lungenventilation. Diese führt zu einer Steigerung der Sauerstoffmenge und damit des Sauerstoffdrucks in den Lungenbläschen. Dadureh wird die Sauerstoffaufnahme des die LungengefäBbe durchströmenden Blutes und damit die den Geweben zur Verfügung stehende Sauerstoffmenge vermehrt. Sie wird weiterhin vermehrt durch Zunahme der Blutfarbstoffmenge, und diese kommt durch $\mathrm{Zu}$ nahme der Zahl der roten Blutzellen zustande. Diese dem Höhenklima eigentümlichen Veränderungen sind vielfach studiert worden und immer wieder bestätigt. Zuerst wurden sie von Tiault festgestellt, der sie, ebenso wie Barcroft und Genossen, beim Aufstieg von Lima auf die Peruanisohen Anden nachweisen konnte. Man wollte, als Viault seine Befunde bekanntgab, nicht recht an deren Wirklichkeit glauben, da man dem Gedanken nicht Raum geben wollte, daß ein klimatischer Einflub eine Konstante, wie es die Zahl der roten Blutzellen ist, ändern sollte, und da eine Anderung der Zellenzahl im Kubikmillimeter Blut - und stets wurde nur diese bestimmt noch nicht beweisend war für eine absolute $\mathrm{Zu}-$ nahme der Gesamtzellenzahl im Körper. Sie könnte auch durch eine nur relative Vermehrung erklärt werden, d. h. durch eine Änderung im Verhältnis von Zellenzahl zu Blutflüssigkeit im Sinne einer Abnahme der letzteren. Jedoch ist schlieblich durch direkte Bestimmung der Gesamthämoglobinmenge im Körper die Tatsache der absoluten Vermehrung sicher erwiesen worden.

So kann es nicht mehr wundernehmen, wenn Barcroft angibt, auch die Veränderung einer anderen Konstante festgestellt zu haben, nämlich eine Anderung in der Bindungsfähigkeit des Hämoglobins für Sauerstoff. Barcroft ermittelte den Sauerstoffdruck in den Lungenbläschen und die im Arterienblute enthaltene Sauerstoffmenge und stellte aus den iso gewonnenen Werten die Dissoziationsspannung des Oxyhämoglobins fest. Es fand sich, daß beim Aufenthalt in der Höhe von Cerro de Pasco das Hämoglobin fähig wurde, bei gleichem Sauerstoffdruck mehr Sauerstoff zu binden als normales Tieflandblut. Diese zweckmäßige Anpassung an die Sauerstoffarmut der Höhenluft ist wichtig, da sioh das Blut beim Durchgang durch die Lungen dort nur unvollkommen mit Sauerstoff sättigt, so daß das Arterienblut nur zu $82 \%$ bei den Fingeborenen, zu $85 \%$ bei den Expeditionsteilnehmern mit Sauerstoff gesättigt zeigte, anstatt wie im Tieflande zu 95-96\% gesättigt zu sein. Damit ist die Grenze eines Sauerstoffmangels also nahegerückt. Bisher wurde von manchen Seiten angenommen, daf eine Sauerstoffsättigung des arteriellen Blutes zu $90 \%$ notwendig sei, um dem Bedarf der Gewebe an Sauerstoff zu genügen.

Utbrigens ist auch nach Untersuchungen von
Krogh und Leitch (Journ. of Physiology 1920) eine andere zweckmäBige Anpassung des Hämoglobins in bezug auf die Sauerstoffaufnahme bei bestimmten Sauerstoffdrucken bekannt. Sie betrifft das Hämoglobin von Fischen, von denen die einen gewöhalich in sauerstoffreichem, die anderen gelegentlich auch in sauerstofformem Wasser leben. Die Dissoziationskurve verläuft bei beiden ganz verschieden. Bei der ersten Gruppe, die also nur ausnahmsweise niedrigen Sauerstoffdrucken ausgesetzt, ist, wie Kabeljau von den Meerfischen, Forelle von den Frischwasserfischen, sättigt sich das Hämoglobin zur Hälfte mit Sauerstoff bei einem $\mathrm{O}_{2}$-Drueke von $18 \mathrm{~mm}$, ganz ähnlich wie bei den Säugern. Bei der zweiten Gruppe jedoch, zu der unter den. Frischwasserfischen Karpfen, Aal, Hecht gehören, sind nur $2-3 \mathrm{~mm}$ $\mathrm{O}_{2}$-Druck erforderlich, um eine Halbsättigung des Hämoglobins mit Sauerstoff zu bewirken. Diese Fischarten sind also bei weitem mehr befähigt, in sauerstoffarmem Wasser zu leben als die erstgenannten.

Die Unterschiede, die individuell in bezug auf den Umfang der Lungenventilation, der Hämoglobinmenge, der Sauerstoffbindung seitens des Hämoglobins bestehen, können die individuellen Verschiedenheiten in der Widerstandsfähigkeit gegen Luftverdünnung verständlich machen.

Barcroft und Genossen untersuchten a'ber noch eine weitere Beziehung, indem sie die von Bohr so genannte Diffusionstionstante der Lunge bei den verschiedenen Expeditionsteilnehmern bestimmten. Bohr bezeichnet mit diesem Ausdrucke die Beziehung zwischen der Menge des in der Minute durch die Lungenoberfläche in das Blut der Lungenkapillaren diffundierenden Sauerstoffes zu dem mittleren Unterschiede des Sauerstoffdruckes, der in den Lungenbläschen und dem Lungenkapillarblute therrseht. Die Differenz zwischen diesen beiden Sauerstoffdruckwerten stellt ja die Triebkraft dar, durch die die Sauerstoffdiffusion durch die Lungenwand geregelt wird. Dabei fand sich nun, daß die Teilnehmer der Expedition in zwei Gruppen geschieden werden konnten. Die der einen zugehörigen hatten eine Diffusionskonstante über 40 , die der anderen unter. 40. Dabei litten die der einen deutlich an verschiedenen Erscheinungen der Bergkrankheit, die der anderen waren davon verschont.

Wie weit dieser Befund für die Vorhersage über Disposition zur Bergkrankheit Bedeutung hat, soll weiter festgestellt werden dadurch, dab bei Ingenieuren, die in die Minendistrikte der Anden gehen wollen, die Diffusionskonstante der Langen zuvor in New York festgestellt wird, und mit diesem Werte die Widerstandsfähigkeit gegen die Bergkrankheit verglichen wird.

Vielleicht werden auf dieser Grundlage die Schwierigkeiten, die sich bis jetzt der Erklärung der Bergkrankheit allein durch Sauerstoffmangel im Einzelfalle entgegenstellen, eine Lösung finden. 